\title{
Formation of intermediate phases and supersaturated solid solution in Al-Cu system during diffusion
}

\author{
A. Rodin* \\ The National University of Science and Technology -"MISiS", Moscow, Russia \\ *Rodin@misis.ru
}

With the use of the methods of optical microscopy, scanning electron microscopy and electron microprobe analysis the studies of diffusion processes in the aluminum - copper system were carried out in the temperature range $320-440{ }^{\circ} \mathrm{C}$. Special attention was paid on the state of the system near $\mathrm{Al} / \mathrm{Cu}$ interface. It was determined that the intermediate phases in the system, corresponding to the equilibrium phase diagram, were not formed at low temperatures. In this case supersaturated solid solution of copper in aluminum could be observed near the interface. Annealing at high temperature leads to the layer-by-layer formation of the equilibrium phases. 